steroid injection are as follows: cross-sectional area 15.68, $15.26,13.53 *, 11.70^{*} \mathrm{~mm}^{2}$; flattening ratio $3.20,3.16,3.12$, $3.10\left({ }^{*} \mathrm{P}<.05\right)$.

Conclusions: As a treatment effect of steroid injection in CTS, clinical improvement precedes morphological change of entrapped median nerve. Ultrasonography using the parameter cross-sectional area of median nerve might be a useful tool for monitoring the improvement of median nerve after CTS injection.

\section{Poster 152}

\section{Ultrasound Imaging of Myofascial Trigger Points and Adjacent Soft Tissue.}

Robin Ortiz, BS (National Institutes of Health, Bethesda, MD); Tadesse M. Gebreab, BS; Naomi Lynn H. Gerber, MD; Jay P. Shah, MD; Siddhartha Sikdar, PhD.

Disclosures: R. Ortiz, None,

Objective: To evaluate an ultrasound (US) imaging scale for visualization and some characterization of myofascial trigger points (MTrPs) and adjacent soft tissue and to assess interrater reliability.

Design: Descriptive study.

Setting: Biomedical research center.

Participants: 16 subjects meeting the Travell and Simons criteria for MTrPs of hypersensitive nodules in a taut band in the upper trapezius.

Interventions: None.

Main Outcome Measures: MTrPs were evaluated by physical examination and US imaging. Sites in each subject were labeled as either active MTrP (spontaneously painful), latent MTrP (nonpainful), or absent MTrP based on physical examination. Vibration sonoelastography (VSE), to assess stiffness, was performed by color Doppler variance imaging, while simultaneously inducing vibrations $(\sim 100 \mathrm{~Hz})$ with a handheld massage vibrator. VSE and grayscale 2D US were analyzed to assign each site a tissue imaging score (TIS) as follows: 0 = uniform echogenicity and stiffness; 1 = focal hypoechoic, stiff nodule; 2 = multiple stiff nodules. Blood flow in the neighborhood of MTrPs was assessed using Doppler imaging. Each site was assigned a blood flow waveform score (BFS) as follows: $0=$ no diastolic flow; $1=$ elevated diastolic flow; 2 = oscillatory and/or retrograde diastolic flow. Two independent raters scored the images.

Results: Our results show that MTrPs appear as focal, hypoechoic regions on 2D US, indicating local changes in tissue echogenicity, and as focal regions of reduced vibration amplitude on VSE, indicating a localized stiff nodule. MTrPs were more likely to have higher TIS and BFS compared with normal muscle $(P<.05$, Mann-Whitney test). Blood flow near MTrPs was retrograde in diastole indicating a highly resistive and compliant vascular bed, consistent with possible blood vessel compression. The BFS score showed a strong intraclass correlation of 0.80 between the 2 raters, while the TIS score showed an intraclass correlation of 0.57.

Conclusions: Current findings demonstrate validity and interrater reliability of our scoring technique for visualizing MTrPs.

\section{Poster 153 \\ Walking Assessment in People With Lumbar Spinal Stenosis: Capacity, Performance, and Self-report Measures.}

Justin Conway, BA (University of Michigan, Ann Arbor, MI); Andrew J. Haig, MD; Christy Tomkins, PhD.

Disclosures: J. Conway, None.

Objective: To examine the relationship between survey instruments, tested walking capacity, and daily ambulatory performance in people diagnosed with lumbar spinal stenosis (LSS).

Design: Prospective laboratory and clinical observational study.

Setting: Tertiary care spine clinic.

Participants: 12 subjects with clinical stenosis significant enough to be scheduled for epidural injection.

Interventions: Subjects filled out functional questionnaires; performed a Self-Paced Walking Test (SPWT) of up to 30 minutes; and wore an Actigraph activity monitor during waking hours for 7 days.

Main Outcome Measures: Questionnaire (including the Swiss Spinal Stenosis Questionnaire, Pain Disability Index, Oswestry Disability Index, Quebec Back Pain Disability Scale, and SF-36), laboratory walk testing (walking capacity) and activity monitors (community ambulation).

Results: There was no statistically significant relationship between walking capacity (SPWT) and community ambulation per day (activity monitors), however the maximum time of continuous activity during community ambulation had a strong relationship $(\mathrm{r}=0.63)$ with the SPWT. Fifteen selfreport measures of ambulation were significantly correlated with the SPWT, activity monitor, or both. Of these, 13 (87\%) were more highly correlated to the SPWT than the activity monitor. The SPWT test had a strong relationship ( $r>.60$, $P<.05$ ) with global function scales, but community ambulation did not.

Conclusions: Ambulation limitation is the hallmark of impairment in LSS. Capacity and performance have been defined as 2 distinct aspects of disability. The results indicate that walking capacity and walking performance in LSS appear to be different constructs. Survey instruments appear to reflect capacity rather than performance. Since increased walking in the community is both a specific desired outcome for stenosis intervention and an important contributor to overall health, this dissociation between walking capacity and performance has implications for the clinical management and outcomes assessment of people with LSS. 